\title{
Empirical Bayes One-side Test for Inverse Exponential Model Based on Negative Associate Samples
}

\author{
Guobing Fan \\ Department of Basic Subjects, Hunan University of Finance and Economics, Changsha, China \\ Email address: \\ fanguobingmath@163.com \\ To cite this article: \\ Guobing Fan. Empirical Bayes One-side Test for Inverse Exponential Model Based on Negative Associate Samples. American Journal of \\ Theoretical and Applied Statistics. Vol. 5, No. 6, 2016, pp. 342-347. doi: 10.11648/j.ajtas.20160506.12
}

Received: September 5, 2016; Accepted: September 23, 2016; Published: October 15, 2016

\begin{abstract}
By using the kernel-type density estimation and empirical distribution function in the case of identically distributed and negatively associated samples, the empirical Bayes one-sided test rules for the parameter of inverse exponential distribution are constructed based on negative associate sample under weighted linear loss function, and the asymptotically optimal property is obtained. It is shown that the convergence rates of the proposed empirical Bayes test rules can arbitrarily close to $\mathrm{O}\left(n^{-1 / 2}\right)$ under suitable conditions.
\end{abstract}

Keywords: Empirical Bayes Test, Negatively Associated Sample, Asymptotic Optimality, Weighted Linear Loss Function, Inverse Exponential Distribution

\section{Introduction}

Empirical Bayes method, first proposed by Robbins [1], is a very important method in statistical inference field, and it has many applications in the field of reliability, lifetime prediction, medical research, ect. [2-6]. Empirical Bayes hypothesis test problem is a hot topic, which attracts many scholars' attention.

For example, Karunamuni [7] discussed the optimal convergence rate of empirical Bayes tests for uniform distributions. Xu et al. [8] investigated the convergence rates of empirical Bayes tests of parameters in one-side truncated distribution families under an asymmetric loss function. Qian and Wei [9] studied the two-sided empirical Bayes test for a class of scale exponential model under a weighed loss function. Chen et al. [10] studied the two-sided empirical Bayes test problem for the continuous one-parameter exponential model with contaminated data by a kernel probability density method. Under Type-II censored samples. Wang et al. [11] studied the empirical Bayes test problem for two-parameter exponential distribution. However, in many situations, such as penetration theory, reliability analysis and multivariable analysis, random samples are probably not independent identical distribution but negatively associated (NA) samples $[12,13]$. For instance, Wei [14] developed a empirical Bayes test of parameter for exponential distribution in the case of NA samples using the linear weighted loss functions. Shi et al. [15] developed a two-sided empirical Bayes test for the parameter of the truncated distribution family in the case of NA samples. Wang and Shi [16] studied the Bayes test problem for a special exponential family under NA samples.

The definition of the NA sequence was first introduced by Esary in [17] as follows:

Definition 1. A sequence of random variables $X_{1}, X_{2}, \ldots, X_{n}$ is said to be a NA sample, if for every pair of disjoint non-empty subsets $A_{1}$ and $A_{2}$ of set $\{1,2, \ldots, n\}$,

$$
\operatorname{Cov}\left(f_{1}\left(X_{i}, i \in A_{1}\right), f_{2}\left(X_{i}, i \in A_{2}\right)\right) \leq 0
$$

Where $f_{1}$ and $f_{2}$ are increasing or decreasing for every independent variable, and their covariance exists. Random variable sequence $\left\{X_{j}, j \in N\right\}$ are said to be NA, if for every natural number $n \geq 2, X_{1}, X_{2}, \ldots, X_{n}$ is negatively associated.

Assume $X$ is a random variable drawn form inverse exponential distribution with the probability density function is 


$$
f(x \mid \theta)=\frac{\theta}{x^{2}} \exp \left(-\frac{\theta}{x}\right), x>0
$$

Here $\theta>0$ is the unknown shape parameter, $\Omega=\{x \mid x>0\} \quad$ is the sample space, and $\Theta=\left\{\theta>0 \mid \int_{\Omega} f(x \mid \theta) d x=1\right\}$ is the parameters space.

Remark 2. It is easily shown that if the random variable $X$ is distributed with exponential distribution, then $X^{-1}$ is distributed with inverse exponential distribution.

The inverse exponential distribution is one of the most important lifetime distributions [18], and the applications in life test, reliability and statistical inference attract many authors' attention. For example, Prakash [19] discussed the Bayes estimation of the inverse exponential model under symmetric and asymmetric loss functions. Rao [20] studied the reliability estimation for the stress-strength model, which the stress and strength are both distributed with inverse exponential distribution. Singh et al. [21] developed a twostage group acceptance sampling plan for a generalized inverse exponential distribution under truncated life test.

In this paper, we will first construct the empirical Bayes test function for testing Eq. (2) in inverse exponential model. Then, we will prove that the proposed test is asymptotically optimal and its convergence rate will also be derived under certain suitable conditions. The remains of this paper is organized as follows: Section 2 will establish a Bayes decision rule for one-side hypothesis test problem. Then Section 3 will put forward a new empirical Bayes decision rule combining the kernel-type density function with empirical distribution function. Finally, Section 4 will give the conclusion of this paper.

\section{Bayes Test}

Given the inverse exponential distribution with probability density function (1), the hypothesis needed to be tested is

$$
H_{0}: \theta \leq \theta_{0} \leftrightarrow H_{1}: \theta>\theta_{0}
$$

Here $\theta_{0}$ is a given constant. This section will construct a new Bayes test rule for hypothesis test problem (2) under the following weighted linear loss functions:

$$
\begin{gathered}
L_{0}\left(\theta, d_{0}\right)=\frac{\theta-\theta_{0}}{\theta} I\left(\theta>\theta_{0}\right) \\
L_{1}\left(\theta, d_{1}\right)=\frac{\theta_{0}-\theta}{\theta} I\left(\theta \leq \theta_{0}\right)
\end{gathered}
$$

Here $d=\left\{d_{0}, d_{1}\right\}$ is the action space, $d_{0}$ indicates accepting $H_{0}, d_{1}$ indicates refusing $H_{0}$.

Remark 1. The weighted linear loss function has many advantages: it is invariant and makes the expression of Bayes test function more concise and makes the empirical Bayes test function easy to construct.

Suppose that $X$ has conditional probability density in Eq. (1) and parameter $\theta$ has the unknown prior distribution function $G(\theta), g(\theta)$ is the corresponding prior probability density function. Then the marginal probability density function of $X$ is:

$$
f_{G}(x)=\int_{\Theta} f(x \mid \theta) d G(\theta)
$$

Let $\delta(x)=P\left(\right.$ accept $\left.H_{0} \mid X=x\right)$ be the randomized decision rule. The Bayes risk function of $\delta(x)$ is

$$
\begin{aligned}
R(\delta(x), G(\theta))= & \int_{\Theta} \int_{\Omega}\left[L_{0}\left(\theta, d_{0}\right) f(x \mid \theta) \delta(x)\right. \\
& \left.+L_{1}\left(\theta, d_{1}\right) f(x \mid \theta)(1-\delta(x))\right] d x d G(\theta)
\end{aligned}
$$

Then

$$
R(\delta(x), G(\theta))=\int_{\Omega} \beta(x) \delta(x) d x+C_{G}
$$

Where $C_{G}=\int_{\Theta} L_{1}\left(\theta, d_{1}\right) d G(\theta)$, and

$$
\beta(x)=\int_{\Theta} \frac{\theta-\theta_{0}}{\theta} f(x \mid \theta) d G(\theta)
$$

According to equation (5), we can get

$$
\begin{gathered}
\beta(x)=\int_{\Theta} f(x \mid \theta) d G(\theta)-\theta_{0} \int_{\Theta} \frac{1}{\theta} f(x \mid \theta) d G(\theta) \\
=f_{G}(x)-\theta_{0} \int_{\Theta} \frac{1}{\theta} f(x \mid \theta) d G(\theta)
\end{gathered}
$$

Define

$$
P_{G}(x)=\int_{\Theta} e^{-\frac{\theta}{x^{2}}} d G(\theta),
$$

then

$$
\beta(x)=f_{G}(x)-x^{-2} \theta_{0} P_{G}(x)
$$

A test is said to be a Bayes test with respect to $G(\theta)$ if $R_{G}=\inf _{\delta} R(\delta(x), G(\theta))$.

Define

$$
\delta_{G}(x)= \begin{cases}1, & \beta(x) \leq 0 \\ 0, & \beta(x)>0\end{cases}
$$

It is easy to show that $\delta_{G}(x)$ is a Bayes test with respect to $G(\theta)$.

The Bayes risk function is: 


$$
R_{G}=\int_{\Omega} \beta(x) \delta_{G}(x) d x+C_{G}
$$

Remark 1. If the prior distribution $G(\theta)$ is known, $\delta(x)$ equals to $\delta_{G}(x)$, we can get $R_{G}$. Unfortunately the $G(\theta)$ is unknown, we need to use the empirical Bayes approach.

\section{Empirical Bayes Test Function}

This section we will construct the empirical Bayes test function. Suppose that $X_{1}, X_{2}, \ldots, X_{n}, X_{n+1}$ are identically distributed and weakly stationary NA sequence. The same probability density function is $f_{G}(x) . X_{1}, X_{2}, \ldots, X_{n}$ are the historical sample, $X_{n+1}$ is the current sample. In this paper, we assume

(A1) $f_{G}(x) \in C_{s, \alpha}, x \in R^{1}$,where $C_{s, \alpha}$ denotes a family of probability density function with $s$ order derivatives and its absolute value not exceeding $\alpha, \alpha$ is a positive integer. $s \geq 2$ is a positive integer.

(A2) Suppose $\mathrm{s} \geq 2$ is an arbitrarily certain natural number, $K_{r}(x)(r=0,1, \ldots s-1)$ is a bounded function of Borel measurable function. When outside of the interval $(0,1)$, their value are zero, and they satisfy the following conditions:

$$
\frac{1}{t !} \int_{0}^{1} y^{t} K_{r}(y) d y=\left\{\begin{array}{l}
1, t=r \\
0, t \neq r, t=0,1,2, \ldots, s-1
\end{array}\right.
$$

Define the Kernel density estimation of $f_{G}(x)$ as:

$$
f_{n}(x)=\frac{1}{n b_{n}} \sum_{\mathrm{j}=1}^{\infty} K_{r}\left(\frac{x-X_{j}}{b_{n}}\right)
$$

Where $\left\{b_{n}\right\}$ is positive integer sequence, and $\lim _{n \rightarrow \infty} b_{n}=0$.

(A3) The covariance of $\left\{X_{n} \mid n \geq 2\right\}$ satisfies condition:

$$
\sum_{\mathrm{j}=1}^{\infty}\left|\operatorname{cov}\left(X_{1}, X_{j}\right)\right| \leq c<\infty
$$

By equations (1) and (8), we can get

$$
\begin{aligned}
E\left[I\left(X_{i}<x\right)\right]=\int_{-\infty}^{x}\left[\int_{\Theta} \frac{\theta}{x^{2}} e^{-\frac{\theta}{x}} d G(\theta)\right] d x \\
\quad=\int_{\Theta}\left[\int_{-\infty}^{x} \frac{\theta}{x^{2}} e^{-\frac{\theta}{x}} d x\right] d G(\theta) \\
=\int_{\Theta}\left[\int_{0}^{x} \frac{\theta}{x^{2}} e^{-\frac{\theta}{x}} d x\right] d G(\theta) \\
=\int_{\Theta}\left[e^{-\frac{\theta}{x}}\right]_{0}^{x} d G(\theta) \\
=P_{G}(x)
\end{aligned}
$$

Hence, the unbiased estimator of $P_{G}(x)$ can be constructed as

$$
P_{n}(x)=\frac{1}{n} \sum_{i=1}^{n} I\left(X_{i}<x\right)
$$

Therefore, the estimator of $\beta(x)$ is

$$
\beta_{n}(x)=f_{n}(x)-x^{-2} \theta_{0} P_{n}(x)
$$

Finally, the empirical Bayes test function could be defined as follows:

$$
\delta_{n}(x)= \begin{cases}1, & \beta_{n}(x) \leq 0 \\ 0, & \beta_{n}(x)>0\end{cases}
$$

Assume $E_{n}$ represents the mean value of the joint distribution of $X_{1}, X_{2}, \ldots, X_{n}$, hence the comprehensive risk of $\delta_{n}(x)$ is

$$
R\left(\delta_{n}(x), G(\theta)\right)=\int_{\Omega} \beta(x) E_{n}\left[\delta_{n}(x)\right] d x+C_{G}
$$

Definition 1. If $\lim _{n \rightarrow \infty} R\left(\delta_{n}(x), G(\theta)\right)=R_{G}$, then the random variable sequence $\left\{\delta_{n}(x)\right\}$ is called asymptotic optimality empirical Bayes test functions.

Definition 2. If $R\left(\delta_{n}(x), G(\theta)\right)-R_{G}=\mathrm{O}\left(n^{-q}\right), q>0$,

Then we say the convergence rate of the test function sequence $\left\{\delta_{n}(x)\right\}$ is $\mathrm{O}\left(n^{-q}\right)$.

By Definitions 1 and 2, we conclude that the optimal evaluation of the empirical Bayes test functions depends on the degree of its risk approximation Bayes risk.

In this paper, we let $c, c_{1}, c_{2}, \ldots$ denote different positive constants in different cases even in the same expression. In order to prove Theorems 1 and 2, which will be illustrated in Section 4, we need to present the following lemmas:

Lemma 1[22] Suppose $R_{G}$ and $R\left(\delta_{n}(x), G(\theta)\right)$ definite in equation (11) and equation (16) separately, then

$$
\begin{aligned}
0 & \leq R\left(\delta_{n}(x), G\right)-R_{G} \\
& \leq \int_{\Omega}|\beta(x)| P\left(\left|\beta_{n}(x)-\beta(x)\right| \geq|\beta(x)|\right) d x
\end{aligned}
$$

Lemma 2 [23] Suppose $f_{n}(x)$ definite as (13), where $X_{1}, X_{2}, \ldots$ are identically distributed and weakly stationary NA sequence. Assume both (A1)- (A3) hold, for $\forall x \in \Omega$

(1) If $f_{G}(x)$ is continuous for $x$, when $\lim _{n \rightarrow \infty} b_{n}=0$ and $\lim _{n \rightarrow \infty} n b_{n}=\infty$ hold, then

$$
\lim _{n \rightarrow \infty} E_{n}\left|f_{n}(x)-f_{G}(x)\right|^{2}=0
$$

(2) If $f_{G}(x) \in C_{s, \alpha}$, let $b_{n}=n^{-\frac{1}{4+2 s}}$, for $0<\lambda \leq 1$, we have 


$$
E_{n}\left|f_{n}(x)-f_{G}(x)\right|^{2 \lambda} \leq c \cdot n^{-\frac{\lambda s}{s+2}}
$$

Lemma 3 Suppose $P_{G}(x)$ and $P_{n}(x)$ definite in equation (8) and equation (13) separately, $X_{1}, X_{2}, \ldots$ are identically distributed and weakly stationary NA sequence, for $0<\lambda \leq 1$, we have

$$
E_{n}\left|P_{n}(x)-P_{G}(x)\right|^{2 \lambda} \leq n^{-\lambda}
$$

Proof. According to equation (14), we have

$$
\begin{aligned}
& E\left(P_{n}(x)-P_{G}(x)\right)^{2 \lambda} \\
& =E\left\{\left[P_{n}(x)-P_{G}(x)\right]^{2}\right\}^{\lambda} \\
& \leq\left[\operatorname{Var}\left(P_{n}(x)\right)\right]^{\lambda}
\end{aligned}
$$

Where

$$
\begin{aligned}
& \operatorname{Var}\left(P_{n}(x)\right)=E\left\{\frac{1}{n} \sum_{i=1}^{n}\left[I\left(X_{i}<x\right)-P_{G}(x)\right]\right\}^{2} \\
& =\frac{1}{n^{2}} \sum_{i=1}^{n} \operatorname{Var}\left[I\left(X_{i}<x\right)\right] \\
& \quad+\frac{2}{n^{2}} \sum_{1 \leq i<j \leq n} \operatorname{Cov}\left(I\left(X_{i}<x\right), I\left(X_{j}<x\right)\right) \\
& =Q_{1}+Q_{2}
\end{aligned}
$$

Because $I\left(X_{i}<x\right)$ is a decreasing about $X_{i}$, and $X_{1}, X_{2}, \ldots$ are identically distributed and weakly stationary NA sequence.

Then

$$
\frac{2}{n^{2}} \sum_{1 \leq i<j \leq n} \operatorname{Cov}\left(I\left(X_{i}<x\right), I\left(X_{j}<x\right)\right) \leq 0
$$

Thus we have

$$
\begin{aligned}
\operatorname{Var}\left(P_{n}(x)\right) & =Q_{1}+Q_{2} \\
& \leq Q_{1} \\
& =\frac{1}{n^{2}} \sum_{i=1}^{n} \operatorname{Var}\left[I\left(X_{i}<x\right)\right] \\
& =\frac{1}{n} \operatorname{Var}\left[I\left(X_{1}<x\right)\right] \\
& =\frac{1}{n}\left\{E\left[I^{2}\left(X_{1}<x\right)\right]-\left[E\left(I\left(X_{1}<x\right)\right)\right]^{2}\right\} \\
\leq & \frac{1}{n}
\end{aligned}
$$

Hence, we have

$$
\begin{aligned}
& E_{n}\left|P_{n}(x)-P_{G}(x)\right|^{2 \lambda} \\
& \leq\left\{E_{n}\left[P_{n}(x)-P_{G}(x)\right]^{2}\right\}^{\lambda} \\
& \leq n^{-\lambda}
\end{aligned}
$$

for any $0<\lambda \leq 1$. The proof of Lemma 3 is completed.

\section{Asymptotic Optimality of Empirical Bayes Test}

Theorem 1. Suppose $\delta_{n}(x)$ be the empirical Bayes test function defined in equation (16), $X_{1}, X_{2}, \ldots$ are identically distributed and weakly stationary NA sequence, when (A1)(A3) hold, if

(1) $\left\{b_{n}\right\}$ is a positive sequence, and satisfy $\lim _{n \rightarrow \infty} b_{n}=0$, $\lim _{n \rightarrow \infty} n b_{n}=\infty$

(2) $\int_{\Theta} \frac{1}{\theta} d G(\theta)<\infty$

(3) $f_{G}(x)$ is continuous about $x$

Then $\lim _{n \rightarrow \infty} R\left(\delta_{n}(x), G(\theta)\right)=R_{G}$

Proof. Let $Q_{n}(x)=|\beta(x)| P\left(\left|\beta_{n}(x)-\beta(x)\right| \geq|\beta(x)|\right)$,

Then $Q_{n}(x) \leq|\beta(x)|$.

Apply (5) and Fubini theorem, we have

$$
\begin{aligned}
\int_{\Omega}|\beta(x)| d x & \leq \int_{\Omega} f_{G}(x) d x+\left|\theta_{0}\right| \int_{\Omega} \frac{1}{\Theta} f(x \mid \theta) d G(\theta) d x \\
& \leq 1+\left|\theta_{0}\right| \int_{\Theta} \frac{1}{\theta} \int_{\Omega} f(x \mid \theta) d x d G(\theta) \\
& =1+\left|\theta_{0}\right| \int_{\Theta} \frac{1}{\theta} d G(\theta)<\infty
\end{aligned}
$$

Then by Lemma 1 and dominated convergence theorem, we have

$$
0 \leq \lim _{n \rightarrow \infty} R\left(\delta_{n}(x), G(\theta)\right)-R_{G} \leq \int_{\Omega} \lim _{n \rightarrow \infty} Q_{n}(x) d x
$$

According to formulas (8) and (13), then by Markov and Jensen inequality, we have

$$
\begin{aligned}
& Q_{n}(x) \leq E_{n}\left|\beta_{n}(x)-\beta(x)\right| \\
\leq & E_{n}\left|f_{n}(x)-f_{G}(x)\right| \\
& +\left|\theta_{0} x^{-2}\right| E_{n}\left|P_{n}(x)-P_{G}(x)\right| \\
\leq & {\left[E_{n}\left|f_{n}(x)-f_{G}(x)\right|^{2}\right]^{\frac{1}{2}} } \\
+ & \left|\theta_{0} x^{-2}\right|\left[E_{n}\left|P_{n}(x)-P_{G}(x)\right|^{2}\right]^{\frac{1}{2}}
\end{aligned}
$$

Then by lemma 2 and lemma 3 , for $\forall x \in \Omega$, we have 


$$
\begin{aligned}
0 & \leq \lim _{n \rightarrow \infty} Q_{n}(x) \leq\left[\lim _{n \rightarrow \infty} E_{n}\left|f_{n}(x)-f_{G}(x)\right|^{2}\right]^{\frac{1}{2}} \\
& +\left|\theta_{0} x^{-2}\right|\left[\lim _{n \rightarrow \infty} E_{n}\left|P_{n}(x)-P_{G}(x)\right|^{2}\right]^{\frac{1}{2}}=0
\end{aligned}
$$

Combining (19) with (20), the theorem is proved.

Theorem 2 Suppose $\delta_{n}(x)$ be the empirical Bayes test function defined in equation (16), $X_{1}, X_{2}, \ldots$ are identically distributed and weakly stationary NA sequence. Assume (A1) -(A3) hold, $f_{G}(x) \in C_{s, \alpha}, 0<\lambda<1$ and $s \geq 2$ is the positive integer, and it satisfies the following conditions:

$$
\begin{gathered}
\text { (B1) } \int_{\Omega}|\beta(x)|^{1-\lambda} d x<\infty \\
\text { (B2) } \int_{\Omega}|\beta(x)|^{1-\lambda}\left|x^{-2}\right|^{\lambda} d x<\infty
\end{gathered}
$$

Then with the choice of $b_{n}=n^{-\frac{1}{2 s+1}}$, we have

$$
R\left(\delta_{n}, G\right)-R_{G}=O\left(n^{-\frac{\lambda s}{2 s+1}}\right)
$$

Proof. First, we can easily get the following result:

$$
\begin{aligned}
& P\left(\left|\beta_{n}(x)-\beta(x)\right| \geq|\beta(x)|\right) \\
= & \int_{\left|\beta_{n}(x)-\beta(x)\right| \geq|\beta(x)|} f(x \mid \theta) d G(\theta) \\
\leq & \int_{\left|\beta_{n}(x)-\beta(x)\right| \geq|\beta(x)|} \frac{\left|\beta_{n}(x)-\beta(x)\right|^{\lambda}}{|\beta(x)|^{\lambda}} f(x \mid \theta) d G(\theta) \\
\leq & \int_{\Omega} \frac{\left|\beta_{n}(x)-\beta(x)\right|^{\lambda}}{|\beta(x)|^{\lambda}} f(x \mid \theta) d G(\theta) \\
= & |\beta(x)|^{-\lambda} E\left|\beta_{n}(x)-\beta(x)\right|^{\lambda}
\end{aligned}
$$

Apply lemma 1 and Markov inequality, we conclude that

$$
\begin{gathered}
0 \leq R\left(\delta_{n}, G\right)-R_{G} \\
\leq \int_{\Omega}|\beta(x)| P\left(\left|\beta_{n}(x)-\beta(x)\right| \geq|\beta(x)|\right) d x \\
\leq \int_{\Omega}|\beta(x)|^{1-\lambda} E_{n}\left|\beta_{n}(x)-\beta_{G}(x)\right|^{\lambda} d x \\
\leq c_{1} \int_{\Omega}|\beta(x)|^{1-\lambda} E_{n}\left|f_{n}(x)-f_{G}(x)\right|^{\lambda} d x \\
+c_{2} \int_{\Omega}|\beta(x)|^{1-\lambda}\left|x^{-2}\right|^{\lambda} E_{n}\left|P_{n}(x)-P_{G}(x)\right|^{\lambda} d x \\
=A_{n}+B_{n}
\end{gathered}
$$

Where $A_{n}=c_{1} \int_{\Omega}|\beta(x)|^{1-\lambda} E_{n}\left|f_{n}(x)-f_{G}(x)\right|^{\lambda} d x$,

$$
B_{n}=c_{2} \int_{\Omega}|\beta(x)|^{1-\lambda}\left|x^{-3}\right|^{\lambda} E_{n}\left|P_{n}(x)-P_{G}(x)\right|^{\lambda} d x
$$

By Lemma 2 and Lemma 3 and condition (B1),we have

$$
\begin{gathered}
A_{n} \leq c_{1} n^{-\frac{\lambda s}{2 s+1}} \int_{\Omega}|\beta(x)|^{1-\lambda}\left|\theta_{0}\right|^{\lambda} d x \leq c_{3} n^{-\frac{\lambda s}{2 s+1}} \\
B_{n} \leq c_{2} n^{-\frac{\lambda}{2}} \int_{\Omega}|\beta(x)|^{1-\lambda}\left|x^{-2}\right|^{\lambda} d x \leq c_{4} n^{-\frac{\lambda}{2}}
\end{gathered}
$$

Then $0 \leq R\left(\delta_{n}, G\right)-R_{G} \leq c_{3} n^{-\frac{\lambda s}{2 s+1}}+c_{4} n^{-\frac{\lambda}{2}}$

Finally it shows that

$$
R\left(\delta_{n}, G\right)-R_{G}=O\left(n^{-\frac{\lambda s}{2 s+1}}\right) .
$$

Remark 2. From Theorems 1 to 2, we know that the new established empirical Bayes test function is asymptotically optimal and its convergence rates $\mathrm{O}\left(n^{-\frac{\lambda s}{2 s+1}}\right)$ can arbitrarily close to $\mathrm{O}\left(n^{-1 / 2}\right)$ under suitable conditions. For positive associate (PA) random variables sequences, we can also use the similar method to construct the empirical Bayes test function and prove the asymptotic performance of the empirical Bayes test function.

\section{Conflict of Interest}

The author has declared that no conflict of interests exists.

\section{Acknowledgement}

This study is partially supported by Natural Science Foundation of Hunan Province (No. 2016JJ4012 and No. 2015JJ3030) and Foundation of Hunan Educational Committee (No.15C0228). The author also gratefully acknowledge the helpful comments and suggestions of the reviewers, which have improved the presentation.

\section{References}

[1] Robbins H., 1956. An empirical Bayes approach to statistics. Annals of Mathematical Statistics, 35(1): 1-20.

[2] Karunamuni R. J., 1996. Empirical Bayes sequential estimation for exponential families: the untruncated component. Annals of the Institute of Statistical Mathematics, 48(4): 711-730.

[3] Phipson B., Lee S., Majewski I. J. Alexander W. S., Smyth G. K., 2013. Empirical Bayes in the presence of exceptional cases, with application to microarray data. Phytochemistry, 26(8): 2247-2250.

[4] Prakash G., 2015. Reliability performances based on empirical Bayes censored Gompertz data. International Journal of Advanced Research, 3(11):1297-1307.

[5] Coram M., Candille S., Duan, Q., Chan K. H., Li Y., Kooperberg C., et al., 2015. Leveraging multi-ethnic evidence for mapping complex traits in minority populations: an empirical Bayes approach. American Journal of Human Genetics, 96(5): 740-52. 
[6] Naznin F., Currie G., Sarvi M., Logan D., 2015. An empirical Bayes safety evaluation of tram/streetcar signal and lane priority measures in melbourne. Traffic Injury Prevention, 17(1): 91-97.

[7] Karunamuni, R. J. (1999). Optimal rates of convergence of monotone empirical Bayes tests for uniform distributions. Statistics \& Decisions, 17(1), 63-86.

[8] Xu, Y. S., Xu, Y., \& Shi, Y. M. (2006). Convergence rates of empirical Bayes test for one-side truncation parameters with asymmetric loss functions. International Journal of Pure \& Applied Mathematics, 27(1), 21-29.

[9] Qian Z., Wei L., 2013. The two-sided empirical Bayes test of parameters for scale exponential family under weighed loss function. Journal of University of Science \& Technology of China, 2(2): 156-161.

[10] Chen J., Jin Q., Chen Z., Liu C., 2013. Two-sided empirical Bayes test for the exponential family with contaminated data. Wuhan University Journal of Natural Sciences, 18(6): 466470 .

[11] Wang L., Shi Y. M., Chang P., 2012. Empirical Bayes test for two-parameter exponential distribution under Type-II censored samples. Chinese Quarterly Journal of Mathematics, 27(1): 54-58.

[12] Shao Q. M., 2000. A comparison theorem on moment inequalities between negatively associated and independent random variables. Journal of Theoretical Probability, 13(2): 343-356.

[13] Sung S. H., 2009. An exponential inequality for negatively associated random variables. Electronic Journal of Statistics, 3(1): 171-174.
[14] Wei L., 2000. The empirical Bayes test problem for scale exponential family: in the case of NA samples. Acta Mathematicae Applicatae Sinica, 23(3: 403-412.

[15] Shi Y., Shi X., Yan J., 2005. Two-sided empirical Bayes test for truncation parameter using NA samples. Information Sciences, 173(1-3): 65-74.

[16] Wang L., Shi Y. M., 2008. The empirical Bayes test for one exponential family using NA samples. Journal of Northwest University, 38(4): 523-526.

[17] Esary J. D.,1983. Negative association of random variables, with applications. Annals of Statistics, 11(1): 286-295.

[18] Lin C., Duran B., Lewis T., 1989. Inverted Gamma as a life distribution. Microelectronics and Reliability, 29(4): 619-626.

[19] Rao G. S., 2013. Estimation of reliability in multicomponent stress-strength based on inverse exponential distribution, International Journal of Statistics and Economics, 10(1): 2837.

[20] Prakash G., 2012. Inverted exponential distribution under a Bayesian viewpoint. Journal of Modern Applied Statistical Methods, 11(1):190-202.

[21] Singh S., Tripathi Y. M., Jun C. H., 2015. Sampling plans based on truncated life test for a generalized inverted exponential distribution. Industrial Engineering \& Management Systems, 14(2): 183-195.

[22] Johns, M. V., 1972. Convergence rates for empirical Bayes two-action problems ii. continuous case. Annals of Mathematical Statistics, 43(3): 934-947.

[23] Chen J. Q, Liu C. H., 2008. Empirical Bayes test problem for the parameter of linear exponential distribution. Journal of Systems Science and Mathematical Sciences, 28(5): 616-626. 